\title{
Checklist
}

\section{Annotated checklist of Collembola of Nepal}

\author{
Prem Bahadur Budha ${ }^{1 *}$ (D) | Pratistha Shrestha ${ }^{1}$ (D)
}

${ }^{1}$ Central Department of Zoology, Institute of Science and Technology, Tribhuvan University, Kathmandu, Nepal

*Correspondence: prem.budha@cdz.tu.edu.np

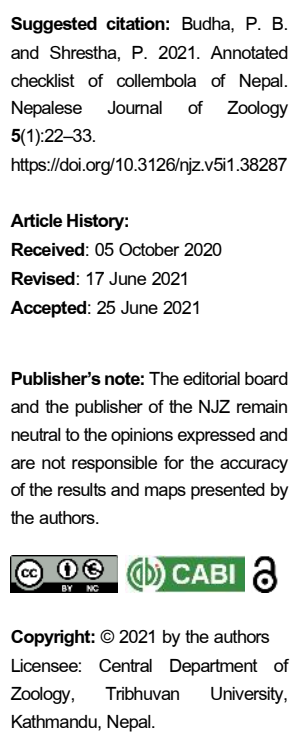

Previously they were considered as insects but the recent molecular studies reveal their pancrustacean relationship (Timmermans et al. 2008). They are the most diversified soil hexapods at local scale $\left(<10 \mathrm{~m}^{2}\right)$ (Deharveng 1996). However, they live in wide range of habitats viz. soil, leaf litter, ground vegetation, tree trunk, tree canopy, seashore, caves, deserts and even in snow or ice (André 1983; Rodgers \& Kitching 1998, 2011; Rusek 1998; Shaw 2013). They play a key role in soil influencing the soil formation, soil microbial ecology, nutrient cycling, and enhance soil fertility by decomposition (Behan-Pelletier 2003; Greenslade 2007; Santos et al. 2008; Snyder \& Callaham 2019). Collembolan short generation period, lower dispersal capacity (Dunger \& Voigtlander 2009), quick recovery with distinct alteration (Frampton 1994; Geissen \& Kampichler 2004), sensitivity (Greenslade 2007) and susceptibility to any kind of disturbances (Petrillo \& Witter 2005; Greenslade et al. 2011) make them valuable marker for the indication of environment (Abbas \& Parwez 2019; Gruss et al. 2019; Yin et al. 2019).

The earliest available record of Nepalese Collembola date backs to early 1910s. The first species known from Nepal was probably Callyntrura lineata (Parona, 1892) which was originally described as Paronella borneri Imms, 1912. After forty six years later, two species Onychiurus himalayensis and $O$. gurjakhanii were described by Choudhuri (1958) from the collection of 1952 British
(Natural History) Museum expedition to Nepal. The major taxonomic contributions on Nepalese Collembola were done only in late 1960s. Yosii (1966a, b, 1971) reported more than 60 species with the description of two new genera viz. Nepalanura and Janetschekbrya and several new species from Nepal. Later new additions were done by Mari Mutt and Bhattacharjee (1980), Mari Mutt (1981), Wilson (1982), Cassagnau (1984, 1993). In subsequent years, new records and species descriptions were further added from the previous expeditions' collections by Bedos and Deharveng (1991), Tamura and Zhao (1997), Thapa (1997), D'Haese and Weiner (1998), Potapov and Cassagnau (2000), Agolin et al. (2009), Thapa (2015), Zhang (2015), Schulz (2018). Thapa (1997) first time complied the all previously known collembolan from Nepal and listed 125 species and however his later published book of Insect Diversity of Nepal comprised only 122 species of Collembola (Thapa 2015).

Most of springtails' inventories in Nepal represent the high Himalaya, with very limited reports from mid-hills and low land. The uppermost record of collembolan from Nepal was from 5800 $m$ asl (Janetschek 1990). Many pristine habitats of different physiographic zones of Nepal are still to be explored. The present list comprises 167 species within 78 genera, 17 families and 17 subfamilies. Taxonomic notes, global distribution of each genus is provided.

\section{2 | Methods}

The present checklist is based on the published papers on Collembola of Nepal including compiled list of Thapa (1997, 2015). All original descriptions were searched from https://biodiversityheritagelibrary.org and https://www.collembola.org. Recent papers were searched in Google and Google scholar. All species data are tabulated with the species name, original 
designation, global species richness and order of taxonomic notes is provided. Classification of lower taxon categories (genus and species) are arranged according to alphabetical order and higher categories are arranged according to (Mandal 2018). The GPS point locality of reported species were noted, if not mentioned in published papers, these data were generated with the locality name given in the paper from the global georeferenced websites- (http://uww.backups.nl/geocoding/, http://www.mapchannels.com/GeocoderSimple.aspx). In many cases, it is difficult to locate the specific point locality for many specific locations in rural areas. In this case, we used only one or two searchable localities for a district for several species. These data were used to prepare distribution map of recorded species of Collembola in Nepal so that district level information may remain intact.

\section{3 | Results and discussion}

\subsection{Collembola species diversity in Nepal}

The updated list of Collembola of Nepal includes 167 species belonging to 78 genera in 17 subfamilies and 17 families (Table 1). Inventory of Collembola was concentrated along the major trekking routes in Nepal particularly west Nepal (Annapurna Conservation Area) and Central Nepal (Langtang area and trekking route from BanepaKavrepalanchowk-Ramechhap-Solukhumbu to Everest base camp) and few high elevation area of west Nepal (Fig. 1). Most of the Tarai and Shiwalik range are completely unexplored with a few exceptions (Fig. 2).

\section{2 | Species Accumulation Curve}

The inventory of Nepalese Collembola began in 1912 . Yosii $(1966,1971)$ discovered 65 species from different part of the country. Janetscheki (1990) is another contributor to describe more than 70 species from Nepal. Based on the available information the species discovery curve is shown in Fig. 3.

\section{3 | Endemic collembolan species}

Endemism patterns varies among regions, habitats and taxa (Deharveng et al. 2008). Endemic species are special attention of conservation point of view. Collembola contain high endemic species (Martin et al. 2000). Nepal is rich in endemic fauna of Collembola. Out of 167 know species 45 species (27\%) are endemic to Nepal (see * mark in the Table 1 for endemic species).

\section{$3.4 \mid$ Codes of references}

[1. Agolin et al. 2009; 2. Bellinger and Ellis 1997; 3. Cassagnau 1984; 4. Cassagnau 1993; 5. Cassagnau and Deharveng 1981;

6. Choudhuri 1958; 7. D'Haese and Weiner 1998; 8. Imms 1912;

9. Janetschek 1990; 10. Mari Mutt 1979; 11. Mari Mutt 1981;
12. Mari Mutt 1985; 13. Mari Mutt and Bhattacharjee 1980; 14. Palacios-Vargas and Gomez-Anaya 1995; 15. Potapov and Cassagnau 2000; 16. Skarzynski and Smolis 2006; 17. Thapa 2015; 18. Wilson 1982; 19. Yosii 1966b; 20. Yosii 1970; 21. Yosii 1971; 22. Yosii 1977; 23. Yosii 1990; 24. Zhang 2015 25. Mandal and Hazra, 2009, 26. Schulz, 2018

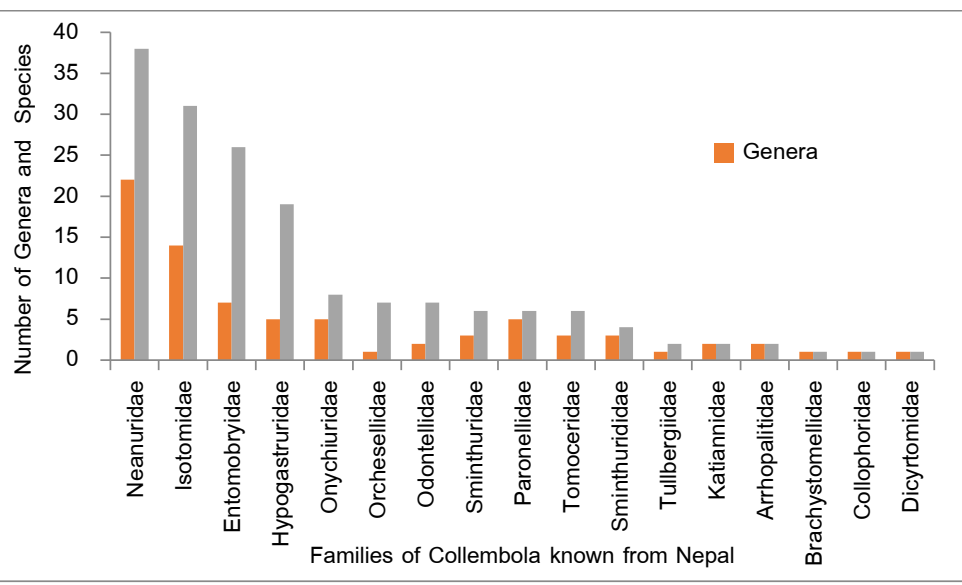

Figure 1. Generic and species diversity within Collembolan families

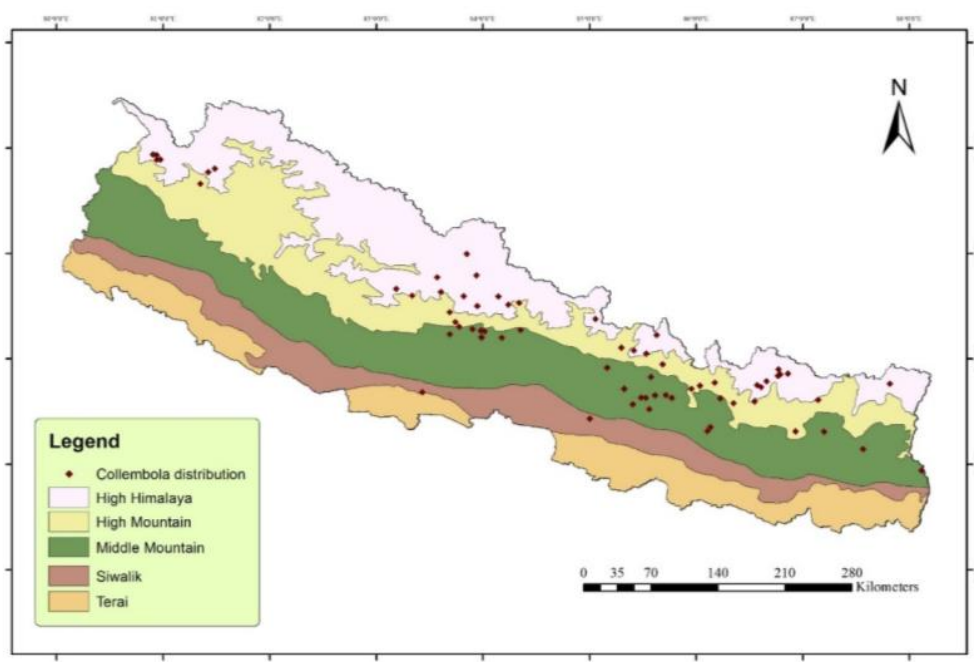

Figure 2. Distribution map of Collembola of Nepal

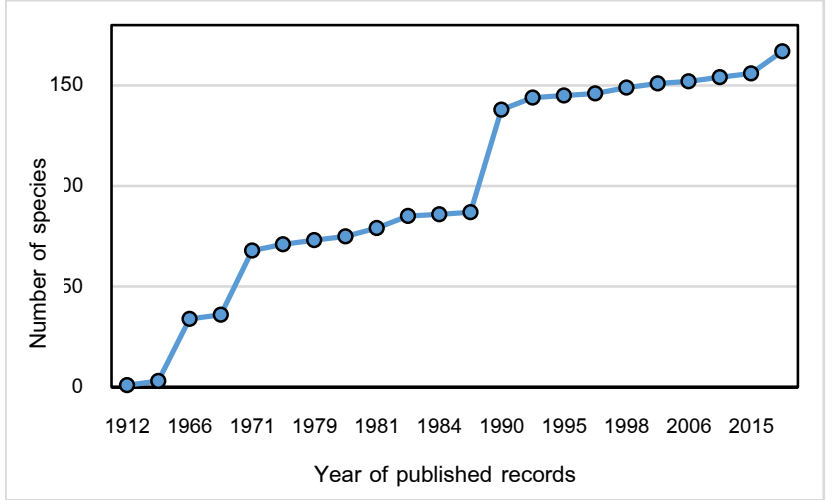

Figure 3. Accumulation curve of the number of Collembola species reported from Nepal 
Table 1. Checklist of the Nepalese Collembola

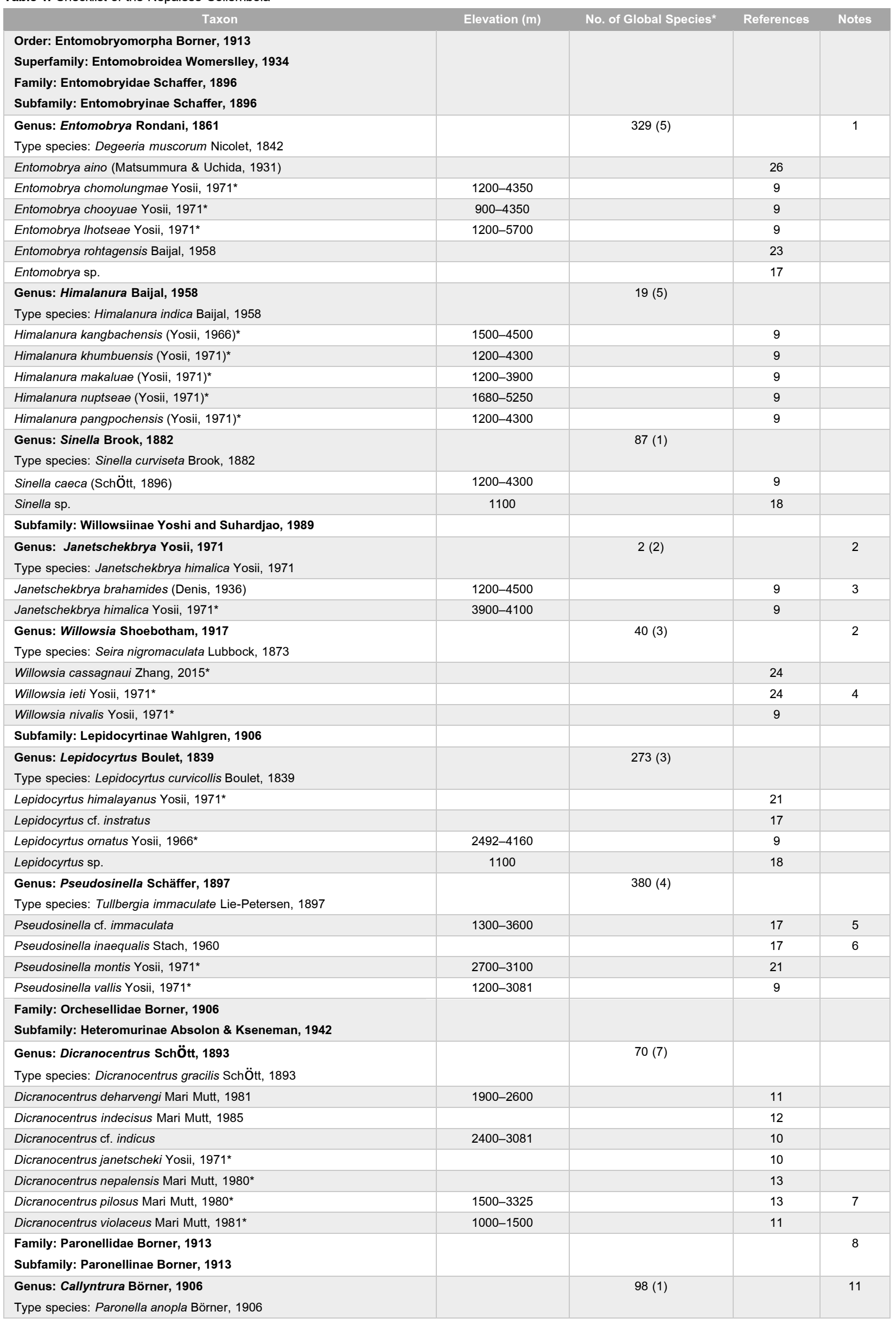


Callyntrura lineata (Parona, 1892) Genus: Cyphoderopsis Carpenter, 1917

Type species: Cyphoderopsis kempi Carpenter, 1917

Cyphoderopsis nepalensis (Wilson, 1982)*

Genus: Cyphoderus Nicolet, 1842

Type species: Cyphoderus albinus Nicolet, 1842

Cyphoderus albinus Nicolet, 1842

Genus Dicranocentroides Imms, 1912

Dicranocentroides flavescens Yosii, 1966

Genus: Troglopedetes Absolon, 1907

Type species: Troglopedetes albus Joseph, 1872

Troglopedetes churchillatus Wilson, 1979*

Troglopedetes nepalensis Wilson, 1982*

Superfamiy: Isotomoidea Szeptycki, 1979

Family: Isotomidae Schaffer, 1896

Subfamily: Anurophorinae Borner, 1901

Genus: Anurophorus Nicolet, 1842

Type species: Anurophorus laricis Nicolet, 1842

Anurophorus cuspidatus Stach, 1920

Anurophorus sp.

Genus: Cryptopygus Willem, 1901

Cryptopygus thermophilus (Axelson, 1900)

Genus: Folsomia Willem, 1902

Type species: Folsomia candida Willem, 1902

Folsomia altamontana Yosii, 1971*

Folsomia candida Willem, 1902

Folsomia diplophthalma (Axelson, 1902)

Folsomia fimetaria (Linnaeus, 1758)

Folsomia obscurocellata Patapov \& Cassagnau, 2000

Folsomia octoculata Handschin, 1925

Folsomia riozoyoshiii Patapov \& Cassagnau, 2000

Genus: Hemisotoma Bagnall, 1949

Type species: Isotoma thermophila Axelson, 1900

Hemisotoma thermophila (Axelson, 1900)

Genus: Isotomiella Bagnall, 1939

Type species: Isotomiella distinguenda Bagnall, 1939

Isotomiella minor (Schäffer, 1896)

Genus: Uzelia Absolon, 1901

Type species: Uzelia setifera Absolon, 1901

Uzelia cf. setifera

Subfamily: Isotominae Schaffer, 1896

Genus: Desoria Agassiz \& Nicolet, 1841

\begin{tabular}{|c|c|c|c|}
\hline & & 8 & 12 \\
\hline & $17(1)$ & & 9 \\
\hline & & 18 & \\
\hline & $72(1)$ & & \\
\hline & & 17 & \\
\hline & & 25 & \\
\hline & $33(1)$ & & 9,10 \\
\hline $1000-1100$ & & 18 & \\
\hline 1100 & & 18 & \\
\hline
\end{tabular}

Type species: Desoria saltans Agassiz \& Nicolet, 1841

Desoria mazda (Yosii, 1971)

Desoria cf. olivacea

Desoria trispinata (Mac Gillivary, 1896)

Genus: Isotoma Bourlet, 1839

Type species: Isotoma viridis Bourlet, 1839

Isotoma anglicana Lubbock, 1873

Isotoma decorata Brown, 1926

Isotoma diverticula Yosii, 1966

Isotoma nepalica Yosii, 1966*

Isotoma cf. viridis

Genus: Parisotoma Bagnall, 1940

Type species: Isotoma notabilis Schäffer, 1896

Parisotoma coeca Yosii, 1966

Parisotoma ekmani (Fjellberg, 1977)

Parisotoma notabilis (Schäffer, 1896)

Genus: Pseudisotoma Handschin, 1924

Type species: Isotoma sensibilis Tullberg, 1876

Pseudisotoma himalayana Yosii, 1971*

Subfamily: Proisotominae Stach, 1947

Genus: Folsomides Stach, 1922

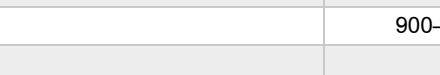

$900-5570$

$52(2)$

-

$202(7)$

\begin{tabular}{|l|l|l|l|}
\hline 1100 & & 18 & 40 \\
\hline & $202(7)$ & & \\
\hline
\end{tabular}

\begin{tabular}{|c|c|}
\hline $900-5600$ & 9 \\
\hline $1200-4300$ & 9 \\
\hline \multirow[t]{5}{*}{$900-5570$} & 9 \\
\hline & 17 \\
\hline & 15 \\
\hline & 22 \\
\hline & 15 \\
\hline
\end{tabular}

$10(1)$

$55(1)$

$1100-3600$

$12(1)$

$1700-4500$

$102(3)$

17

(1)

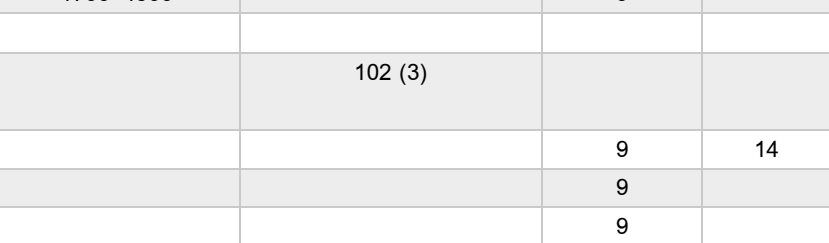

$68(4)$

900-5570

2700-4500

1219-4300

1420-5450

\begin{tabular}{|c|c|c|c|}
\hline & $28(3)$ & & \\
\hline & & 19 & \\
\hline & & 9 & 13 \\
\hline & $8(1)$ & & \\
\hline & & 9 & \\
\hline & & & \\
\hline
\end{tabular}


Type species: Folsomides parvulus Stach, 1922

Folsomides angularis (Axelson, 1905)

Folsomides nepalicus Yosii, 1971*

Folsomides parvulus Stach, 1922

Genus: Folsomina Denis, 1931

Type species: Folsomina onychiurina Denis, 1931

Folsomina onychiurina Denis, 1931

Genus: Proisotoma Börner, 1901

Type species: Isotoma minuta Tullberg, 1871

Proisotoma tenella (Reuter, 1895)

Genus: Weberacantha Christiansen, 1951

Type species: Weberacantha octa Christiansen, 1951

Weberacantha janetscheki (Yosii, 1971)

Superfamily: Tomoceroidea Szeptycki, 1979

Family: Tomoceridae Schaffer, 1896

Genus: Plutomurus Yosii, 1956

Plutomurus vigintiferispina Lee, 1974

Genus: Tomocerina Yosii, 1955

Type species: Tomocerus minutus Tullberg, 1876

Tomocerina simplex Yosii, 1966

Tomocerina aokii (Yosii, 1972)

Genus: Tomocerus Nicolet, 1842

Type species: Pogonognathellus longicornis Muller, 1776

Tomocerus nepalicus Yosii, 1971*

Tomocerus ocreatus Denis, 1948

Tomocerus steinbocki Yosii, 1971

$1200-5570$

$1100-4500$

\begin{tabular}{|l|l|l|l|}
\hline & $5(1)$ & 9,18 & 41 \\
\hline & & & \\
\hline & $78(1)$ & 17 & 15 \\
\hline
\end{tabular}

Order: Poduromorpha Borner, 1913

Superfamily: Hypogastruroidea Salmon, 1964

Family: Hypogastruridae Borner, 1906

Genus: Acherontides Bonet, 1945

Type species: Acherontides atoyacensis Bonet, 1945

Acherontides edaphicus Yosii, 1971

Genus: Ceratophysella Börner, 1932

Type species: Podura armata Nicolet, 1842

Ceratophysella communis (Folsom, 1898)

Ceratophysella denticulata (Bagnall, 1941)

Ceratophysella horrida (Yosii, 1960)

Ceratophysella planipila Yosii, 1966

Ceratophysella postantennalis Yosii, 1966

Ceratophysella cf. sinensis

Ceratophysella cf. vulgaris

Genus: Hypogastrura Bourlet, 1839

Type species: Hypogastrura aquatic Bourlet, 1839

Hypogastrura carpentana Bonet, 1930

Hypogastrura distincta (Axelson, 1902)

Hypogastrura himalayana Yosii, 1971

Hypogastrura nepalica Yosii, 1966*

Genus: Willemia Börner, 1901

Type species: Willemia anophthalma Börner, 1901

Willemia annapurna D'Haese \& Weiner, 1998

Willemia anophthalma Börner, 1901

Willemia buddenbrocki Huther, 1959

Willemia nepalensis D'Haese \& Weiner, 1998*

Willemia wandae Tamura \& Zhao, 1997

Genus: Xenylla Tullberg, 1869

Type species: Xenylla maritime Tullberg, 1869

Xenylla obscura Imms, 1912

Xenylla yosiiana de Gama, 1971

Xenylla mucronata Alexon, 1903

$78(1)$

7 (1)

9

26

$4(1)$

19

$08(3)$

$600-2400$

$3900-4500$

17

C

Superfamily: Neanuroidea Massoud, 1967

Family: Brachystomellidae Stach, 1949

Genus: Brachystomella Ågren, 1903

12 (1)

$40(7)$

950-2000

2700-2850

$1200-4550$

2400

(1)

$75(3)$

Type species: Brachystomella maritime Ågren, 1903 
Brachystomella parvula (Schäffer, 1896)

Family: Neanuridae Borner, 1901

Subfamily: Frieseinae Massoud, 1967

Genus: Friesea Dalla Torre, 1895

Type species: Triaena mirabilis Tullberg, 1871

Friesea excelsa Denis, 1936

Friesea paula Yosii, 1966

Friesea sublimis Macnamara, 1921

Subfamily: Neanurinae Borner, 1901

Genus: Chaetobella Cassagnau, 1983

Type species: Lobella numatai Yosii, 1966

Chaetobella numatai (Yosii, 1966)

Genus: Gnatholonche Börner, 1906

Type species: Achorutes lipaspis Börner, 1906

Gnatholonche anomala (Yosii, 1966)

Genus: Himalmeria Cassagnau, 1984

Type species: Himalmeria lama Cassagnau, 1984;

Himalmeria digitata Cassagnau, 1984

Himalmeria gurung Cassagnau, 1984*

Himalmeria himalayana (Yosii, 1966)*

Genus: Lobella Cassagnau, 1983

Lobella kraepelini (Börner, 1906)

Genus: Lobellina Yosii, 1956

Type species: Lobella roseola Yosii, 1954

Lobellina roseola (Yosii, 1954)

Genus: Nepalanura Yosii, 1966

Type species: Nepalanura paranuroides Yosii, 1966

Nepalanura paranuroides Yosii, 1966

Genus: Nepalimeria Cassagnau, 1984

Type species: Nepalimeria dal Cassagnau, 1984

Nepalimeria coccinea Cassagnau, 1984

Nepalimeria dal Cassagnau, 1984

Nepalimeria ganesh Cassagnau, 1993*

Nepalimeria heterochaeta Cassagnau, 1984

Nepalimeria khorensis Cassagnau, 1984*

Nepalimeria lepchana (Yosii, 1966)*

Genus: Paleonura Cassagnau, 1982

Type species: Paleonura spectabilis Cassagnau, 1982

Paleonura khumbica (Cassagnau, 1971)*

Paleonura monophthalma (Yosii, 1966)

Paleonura reducta (Yosii, 1966)

Paleonura siva (Yosii, 1966)

Paleonura spectabilis Cassagnau, 1982

Genus: Paranura Axelson, 1902

Type species: Paranura sexpunctata Axelson, 1902

Paranura quadripunctata Yosii, 1966

Paranura ieti (Yosii, 1966)

Genus: Propeanura Yosii, 1956

Type species: Neanura pterothryx Yosii, 1956

Propeanura hygrophila (Cassagnau \& Deharveng, 1981)

Propeanura lapidicola (Cassagnau \& Deharveng, 1981)

Genus: Synmeria Cassagnau, 1983

Type species: Phylliomeria miranda Yosii, 1966

Synmeria miranda (Yosii, 1966)

Genus: Yuukianura Yosii, 1955

Type species: Protanura aphoruroides Yosii, 1953

Yuukianura yasudai (Yosii, 1966)

Subfamily: Pseudachorutinae Borner, 1906

Genus: Cassagnaudina Massoud, 1967

Type species: Pseudachorudina coiffaiti Cassagnau, 1955

Cassagnaudina khumbuensis Yosii,1971*

Genus: Cephalachorutes Bedos \& Deharveng, 1991

Type species: Cephalachorutes asiaticus Bedos \& Deharveng, 1991
2700

$197(3)$

\begin{tabular}{|c|c|c|}
\hline $1200-4550$ & \\
\hline 2700 & \\
2000 & \\
\hline
\end{tabular}

9 (1)

$2400-2705$

$25(1)$

3600

$20(3)$

25

$1(1)$

$2400-2700$

$6(6)$

25

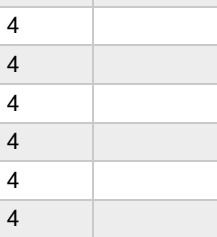

$53(5$

\begin{tabular}{|c|c|c|c|}
\hline $1200-1500$ & 9 & 21,22 \\
\hline 2400 & 19 & \\
\hline & & 19 & 22 \\
\hline & & 19 & \\
\hline
\end{tabular}

$37(2)$

700

$$
19
$$

$14(2)$

5

3 (1)

$10(1)$

23

$19 \quad 24$

4 (1)

$700-4550$

16 (1) 
Cephalachorutes nakaoi (Yosii, 1966)

Genus: Furculanurida Massoud, 1967

Type species: Micranurida africana Massoud, 1963

Furculanurida ashrafi (Yosii, 1966)

Genus: Grananurida Yosii, 1954

Type species: Grananurida tuberculata Yosii, 1954

Grananurida alba (Yosii, 1966)

Genus: Hylaeanura Arlé, 1966

Type species: Paranurella infima Arlé , 1959

Hylaeanura nepalensis (Yosii, 1966)*

Genus: Micranurida Börner, 1901

Type species: Micranurida pygmaea Börner, 1901

Micranurida pygmaea Börner, 1901

Genus: Pseudachorudina Stach, 1949

Type species: Pseudachorudina alpine Stach, 1949

Pseudachorudina nepalica Yosii, 1966*

Genus: Pseudachorutes Tullberg, 1871

Type species: Pseudachorutes subcrassus Tullberg, 1871

Pseudachorutes corticicolus (Schäffer, 1896)

Pseudachorutes kanchenjungae Yosii, 1966*

Genus: Simonachorutes Skarzyniski, Arbia, Piwnik, 2016

Simonachorutes cf. romeroi

Superfamily: Onychiuroidea D’Haese, 2002

Family: Odontellidae Massoud, 1967

Genus: Austrodontella Ellis \& Bellinger, 1973

Type species: Odontella trispina Salmon, 1951

Austrodontella trispina (Womersley, 1935)

Genus: Superodontella Stach, 1949

Type species: Odontella ewingi Folsom, 1916

Superodontella cf. distincta

Superodontella gladiator Agolin, Houssin \& Deharveng, 2009

Superodontella lamellifera (Axelson, 1903)

Superodontella montemaceli Arbea \& Weiner, 1992

Superodontella nepalica (Yosii, 1971) *

Superodontella virgulata Yosii, 1966

Family: Tullbergiidae Bagnall, 1935

Genus: Mesaphorura Börner, 1901

Type species: Mesaphorura krausbaueri Börner, 1901

Mesaphorura himalayensis Yosii, 1971*

3500

19

\begin{tabular}{r|r}
$17(1)$ \\
\hline
\end{tabular}

9

\begin{tabular}{ll} 
(1) & 29 \\
\hline
\end{tabular}

3600

19

\begin{tabular}{|l|r|}
\hline $5(1)$ & 27 \\
\hline
\end{tabular}

\begin{tabular}{|l|l|l|}
\hline 19 & 28 \\
\hline
\end{tabular}

17

$5(1)$

2400

19

\begin{tabular}{c|c|c|} 
& $1200-4550$ & \\
4160 & & \\
\hline
\end{tabular}

931

9

26

Mesaphorura krausbaueri Börner, 1901

Family: Onychiuridae Borner, 1901

Subfamily: Onychiurinae Borner, 1901

Genus: Deharvengiurus Weiner, 1996

Type species: Onychiurus argus Denis, 1924

Deharvengiurus cf. argus

$2(1)$

Genus: Oligaphorura Bagnall, 1949

Type species: Aphorura absoloni Börner, 1901

Oligaphorura cf. groenlandicus

Oligophorura palissai (Yosii, 1971)

Genus: Onychiurus Gervais, 1841

Type species: Podura ambulans Linnaeus, 1758

Onychiurus decemsetosus Yosii, 1966

Genus: Orthonychiurus Stach, 1954

Type species: Onychiurus rectopapilatus Stach, 1933

Orthonychiurus gurjakhanii Choudhuri, 1958*

Orthonychiurus himalayensis (Choudhuri, 1958)*

Genus: Thalassaphorura Bagnall, 1949

Type species: Onychiurus franzi Stach, 1946

Thalassaphorura yodai (Yosii, 1966)

Thalassaphorura cf. encarpata

Order: Symphypleona Borner, 1901

Family: Arrhopalitidae Stach, 1956

Genus: Arrhopalites Börner, 1906

\begin{tabular}{|c|c|c|c|}
\hline & $2(1)$ & & \\
\hline \multirow[t]{2}{*}{$900-4550$} & & 17 & \\
\hline & $70(5)$ & & \\
\hline \multirow[t]{2}{*}{$1400-3600$} & & 17 & 32 \\
\hline & & 1 & \\
\hline $3500-4160$ & & 17 & \\
\hline \multirow[t]{2}{*}{2800} & & 26 & \\
\hline & & 9 & \\
\hline 2400 & & 19 & \\
\hline
\end{tabular}

$200-4300$

$1200-4300$

9

$10(1)$

$62(2)$

17

$50(1)$

$1200-4300$

9

$28(2)$

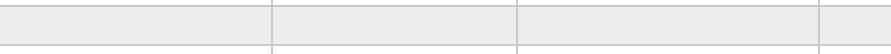

6

$71(2)$

1100

18,22

17

$52(1)$

36 


\begin{tabular}{|c|c|c|c|c|}
\hline \multicolumn{5}{|l|}{ Type species: Sminthurus caecus Tullberg, 1871} \\
\hline Arrhopalites nivalis Yosii, 1966 & $950-4500$ & & 20 & \\
\hline $\begin{array}{l}\text { Genus: Pygmarrhopalites Vargovitsh, } 2009 \\
\text { Type species: Dicyrtoma pygmaea Wankel, } 1860\end{array}$ & & $101(1)$ & & 36,37 \\
\hline Pygmarrhopalites habei (Yosii, 1956) & & & 21 & \\
\hline \multicolumn{5}{|l|}{ Family: Collophoridae Bretfeld, G, 1999} \\
\hline $\begin{array}{l}\text { Genus: Collophora Richards, } 1964 \\
\text { Type species: Arrhopalites quadrioculata Denis, } 1933\end{array}$ & & $9(1)$ & & \\
\hline Collophora mysticiosa Yosii, 1966 & 2000 & & 19 & \\
\hline \multicolumn{5}{|l|}{$\begin{array}{l}\text { Family: Dicyrtomidae Borner, } 1906 \\
\text { Subfamily: Ptenothricinae }\end{array}$} \\
\hline $\begin{array}{l}\text { Genus: Ptenothrix Börner, } 1906 \\
\text { Type species: Podura atra Linnaeus, } 1758\end{array}$ & & $88(1)$ & & \\
\hline Ptenothix himalayensis Yosii, $1966^{*}$ & $2000-3600$ & & 19 & 38 \\
\hline \multicolumn{5}{|l|}{ Family: Katiannidae Borner, 1913} \\
\hline $\begin{array}{l}\text { Genus: Papirinus Yosii, } 1954 \\
\text { Type species: Papirius prodigiosus Yosii, } 1954\end{array}$ & & $4(1)$ & & \\
\hline Papirius ieti Yosii, 1966 & $2000-28000$ & & 20 & \\
\hline $\begin{array}{l}\text { Genus: Stenognagthellus Stach, } 1956 \\
\text { Type species: Stenognagthellus denisi Cassagnau, } 1953\end{array}$ & & $4(1)$ & & 35 \\
\hline Stenognagthellus cassagnaui Yosii, 1966 & 2705 & & 19 & \\
\hline \multicolumn{5}{|l|}{$\begin{array}{l}\text { Family: Sminthuridae Lubbock, } 1862 \\
\text { Subfamily: Sminthurinae Lubbock, } 1862\end{array}$} \\
\hline $\begin{array}{l}\text { Genus: Sminthurus Latreille, } 1802 \\
\text { Type species: Podura viridis Linnaeus, } 1758\end{array}$ & & $83(2)$ & & \\
\hline Sminthurus hispanicus Nayrolles, 1995 & 3400 & & 26 & \\
\hline Sminthurus leucomelanus, Nayrolles, 1995 & 2800 & & 26 & \\
\hline Sminthurus sp.1 & $1500-2675$ & & 9 & \\
\hline Sminthurus sp.2 & $1200-4300$ & & 9 & \\
\hline \multicolumn{5}{|l|}{ Subfamily: Sphyrothecinae Betsch, 1980} \\
\hline $\begin{array}{l}\text { Genus: Sphyrotheca Börner, } 1906 \\
\text { Type species: Sminthurus multifasciata Reuter, } 1881\end{array}$ & & $26(1)$ & & \\
\hline Sphyrotheca gangetica Yosii, 1966 & & & 19 & \\
\hline $\begin{array}{l}\text { Genus: Szeptyckitheca Betsch \& Weiner, } 2009 \\
\text { Type species: Szeptyckitheca kesongenesis Betsch \& Weiner, } 2009\end{array}$ & & $12(1)$ & & 39 \\
\hline Szeptyckitheca nepalica (Yosii, 1966)* & $1360-2750$ & & 19 & \\
\hline \multicolumn{5}{|l|}{ Family: Sminthurididae Borner, 1906} \\
\hline $\begin{array}{l}\text { Genus: Sphaeridia Linnaniemi, } 1912 \\
\text { Type species: Sminthurus pumilis Krausbauer, } 1898\end{array}$ & & $70(2)$ & & \\
\hline Sphaeridia murphyi Yosii, 1966 & 2750 & & 19 & \\
\hline Sphaeridia zaheri Yosii, 1966 & & & 19 & \\
\hline $\begin{array}{l}\text { Genus: Stenacidia Börner, } 1906 \\
\text { Type species: Sminthurides violacea Salmon,1956 }\end{array}$ & & $3(1)$ & & \\
\hline Stenacidia picta Yosii, 1966 & $1200-3600$ & & 9 & \\
\hline $\begin{array}{l}\text { Genus: Yosiides Massoud \& Betsch, } 1972 \\
\text { Type species: Sminthurides himachal Yosii, } 1966\end{array}$ & & $2(1)$ & & \\
\hline Yosiides himachal (Yosii, 1966) & 2705 & & 19 & \\
\hline
\end{tabular}

\section{5 | Annotations}

1. Species level identification of the Genus Entomobrya Rondani, 1861 is considered the problematic due to intraspecific morphological variations. Combination of both chaetotaxy and morphological characters are useful tools to identify them (Jordana \& Baquero 2005), but it is not without complications (Katz et al. 2015).

2. Willowsia and Janetschekbrya are included in Willowsia-complex because of their scale morphology and chaetotaxy, directly derived from the Himalanura-like species (Zhang et al. 2011).

3. Sira brahmides and its re-described combination Janetschekbya brahmides are proposed to be different species. It is supported by the differences in claw structure, color and geographical distribution (Zhang et al. 2011).

4. Zhang (2015) re-described Willowsia ieti Yosii, 1971 with the addition of characteristics such as details of mouthparts and whole body chaetotaxy.

5. Folsom (1902) stated formerly that Pseudosinella argentae differs from Tullbergia immaculata in claw and mucrones but these two species are now synonymized (Bellinger et al. 1996-2021).

6. Pseudosinella inaequalis Bagnall, 1941 and Pseudosinella inaequalis Stach, 1960 nec Bagnall are treated as two different species (Christiansen \& Bellinger 1996, Bellinger et al. 1996-2021).

7. Dicranocentrus pilosus Mari Mutt, 1980 has distinct morphology than other members of the genus Dicranocentrus, so it should be revised and placed in another genus (Cipola et al. 2016) 
8. Cyphoderidae is included as subfamily within Paronellidae (SotoAdames et al. 2008).

9. Absolon (1907) mentioned the type specie of Troglopedetes was as T. pallidus Absolon, 1907 (Wilson 1982, Thibaud \& Najt 1988).

10. Troglopedetes Absolon, 1907 was synonymized with Troglopedetina Delamare Deboutteville, 1945 after reviewing correspondence of characters) and Troglopedetes Absolon, 1907 might be junior homonym or synonym to Troglopedetes Joseph, 1872 (Ellis \& Bellinger 1973, Wilson 1982).

11. Paronella anopla is synonymized with Callyntrura longicornis (Mitra \& Dallai 1980, Bellinger et al. 1996-2021)

12. Yosii (1966a) transferred the species Paronella borneri Imms, 1912 and Hanschinphysa borneri Salmon, 1966 to Callyntrura and synonymized with his species. The species Paronella borneri is also synonymized with Callyntrura lineata (Parona, 1892) (Mandal \& Hazra 2009).

13. As Parisotoma ekmani (Fjellberg, 1977) have lost microsensilla on $2^{\text {nd }}$ abdominal segment is group position in current taxonomy is uncertain (Potapov et al. 2011).

14. Isotoma mazda Yosii, 1971 was transferred to the genus Desoria despite its unusual fit as finding new characters for new taxon was not possible (Yosii, 1990). Mandal \& Hazra (2009) placed this species under the subgenus Desoria of the genus Isotoma.

15. Lawrence (1969) re-described Folsomina onychiurina Denis, 1931 and stated that some specimens described from Nepal may refer to F. yosii. However, Christiansen \& Bellinger (1992) clarified that both are certainly the same species. And, the species described by Lawrence (1969) is undoubtedly a different species which was later named as Folsomina lawrencei by Greenslade (1999).

16. Gender of the type species of genus Acherontides is treated as neuter in original description (Ellis \& Bellinger 1973).

17. Ceratophysella postantennalis recorded by Yosii (1966b) from Solukhumbu match the description of Ceratophysella morula, so the morphologies of both should be well studied before considering the former species as new (Skarzynski \& Smolis 2006).

18. Ceratophysella postanetennalis and Hypogastrura nepalica are diagnosed as conspecific thus synonymized. It is because the original description of Ceratophysella postantennalis was based on the Hypogastrura nepalica (See Skarzynski \& Smolis 2006).

19. Yosii $(1960,1962)$ split the genus Hypogastrura into three subgenus viz. Ceratophysella, Cyclograna and Hypogastrura based on the known chaetotaxy, later treated as genus. But he mentioned in the paper that his description of genus Hypogastrura resembles with some members of genus Ceratophysella so it needs further modifications (Yosii 1960).

20. Willemia buddenbrocki-group is monophyletic and has distinct clade of its 10 species involved (D'Haese \& Weiner 1998).

21. Name of the author of Paleonura khumbica is given as Paleonura khumbica (Yosii, 1971) in Janetschek (1990) and Paleonura khumbica (Cassagnau, 1971) in Bellinger et al. (1996-2021).

22. Janetschek (1990) has incorrect spelling for Paleonura as Paleanura.

23. Yosii (1977) had established Lobellina as subgenus and Yuukianura as special group within the genus Lobella.

24. Deharveng et al. (2017) proposed for the transfer of Lobella yasudai to Genus Yuukianura for their poorly developed tubercles, complex mouthparts and lateral shift of chaetae on $5^{\text {th }}$ abdominal segment.
25. Cassagnau (1993) clarified that there is an adaptive convergence between the Himalmeria and Nepalimeria which is why they were commonly placed in the genus Phylliomeria formerly by Yosii.

26. Selection of Lobella ieti Yosii, 1966 as type species for Propanura by Cassagnau in 1980 violates the article 61 of International Code of Zoological Nomenclature (Ellis \& Bellinger 1984).

27. The species Paranurella infima was first described by Arlé in 1959 (Vázquez et al. 1998) and not in 1960. Genus Hylaeanura Arlé, 1966, also considered as Kenyura by other authors previously, is delineated based on hypertrophied sensilla $S 8$ on $4^{\text {th }}$ antennal segment (Vázquez et al. 1998, Palacios-Vergas \& Deharveng 2010).

28. Paranura nepalensis Yosii, 1966 was considered to be included in the genus Kenyura but as the S8 sensillum is hypertrophied it was transferred to the genus Hylaeanura (Vázquez et al. 1998)

29. Agranurida Kim and Lee, 2000 referred to as synonymy of the genus Grananurida is actually incorrectly spelled (Bellinger et al. 19962021).

30. Pseudachorutella nakaoi Yosii, 1966 was transferred to the genus Cephalachorutella primarily based on the antennal chaetotaxy (Bedos \& Deharveng 1991).

31. Bellinger et al. (1996-2021) has incorrectly mentioned date of description of Pseudachorutes corticicolus as 1897 instead of 1896.

32. Odontella distincta is listed in Checklist of Collembola of the World as Odontella distincta Peja, 1985 nec Yosii, 1954 (Bellinger et al. 1996-2021).

33. Mesaphorura himalayensis Yosii, 1971 should be revised for its genus as its pseudocelli are not star shaped (Bellinger et al. 1996-2021).

34. Thalassaphorura encarpata (Denis, 1931) is synonymized with Onychiurus hortensis Gisin, 1949 (Bellinger et al. 1996-2021)

35. Stenognathellus Cassagnau, 1953 is an unavailable name as type species is not designated for it (Ellis \& Bellinger 1973).

36. With the revision, species of the genus Arrhopalites was split into two genera as caecus-group and pygmaeus-group into Arrhopalites and Pygmarrhopalites respectively (Vargovitsh 2009)

37. Zeppelini (2011) discusses that genus Pygmarrhopalites only informs about the pygmaeus-group so it should be treated as junior synonym of Arrhoplaites.

38. Yosii (1969) re-described S-chaetotaxy of Ptenothrix himalayensis to free the ambiguity of previous description.

39. All the species of the genus Szeptyckitheca are to be reexamined of body chaetotaxy for clear definition of genus and its phylogenetic relationship (Zeppelini et al. 2019).

40. Wilson (1982) misspelled "Cryptopygus" as "Cryptopagus".

41. Wilson (1982) identified Folsomides exiguss from Mahendra cave, Pokhara which is a junior synonym of Folsomides parvulus.

42. Wilson (1982) found Lobella kraepelina from Mahendra cave, Pokhara. But other authors spelled it as "kraepelini" (Yosii, 1959; Mandal and Hazra, 2009). Mandal (2018) placed this species under the genus Hyperlobella Cassagnau, 1988

\section{5 | Conclusions}

The history of collembolan research in Nepal is of about 110 years. All collembolan faunae of Nepal were investigated by foreign scientists. Species level information is scattered in various publications all over the world. Species checklists are important to understand species diversity and richness in a particular geographic area given in specific time which is the first 
basic information required for ecological studies, biodiversity assessments and developing biodiversity conservation strategies. Faunal checklists are lacking in Nepal. The present list includes 167 species belonging to 78 genera and 17 families including 45 endemic species to Nepal. The most of the collections of collembolan was found from the mountain trekking routes in Nepal. Tarai and Shiwalik range remains still unexplored.

\section{Acknowledgements}

We would like to acknowledge Prof. Dr. V. K. Thapa for his initiation to compile the data on insects of Nepal and provided all past literature to PB including his book published in 2015. We also acknowledge anonymous reviewers for their valuable comments which helped to improve the paper quality.

\section{Authors' contributions}

Both authors wrote the manuscript. Shrestha, P. prepared the distribution map of Collembola in Nepal. Budha, P. edited the manuscript, incorporated reviewer's comments and finalized it.

\section{Conflicts of interest}

Authors declare no conflict of interest.

\section{ORCID}

\section{Prem Bahadur Budha (iD https://orcid.org/0000-0003-0205-0979 Pratistha Shrestha (D) https://orcid.org/0000-0002-7164-7210}

\section{References}

Abbas, M. J. and Parwez, H. 2019. Diversity and relative abundance of Collembola in a wheat (Triticum aestivum) field at Aligarh. bioRxiv. https://doi.org/https://doi.org/10.1101/580811.

Agolin, M., Houssin, C. and Deharveng, L. 2009. Superodontella gladiator, a new species of the family Odontellidae (Collembola: Poduromorpha) from Nepal with extremely elongated mouthparts. Zootaxa 2208:51-57. https://doi.org/10.11646/zootaxa.2208.1.4

André, H. 1983. Notes on the ecology of corticolous epiphyte dwellers. 2. Collembola. Pedobiologia. https://doi.org/10.1016/b978-0-12592201-2.50078-6

Bedos, A. and Deharveng, L. 1991. Cephalachorutes gen. n., a new genus of tropical Neanuridae (Collembola). Tijdschrift voor Entomologie 134(1):145-153

Behan-Pelletier, V. M. 2003. Acari and Collembola biodiversity in Canadian agricultural soils. Canadian Journal of Soil Science 83:279-288. https://doi.org/10.4141/s01-063

Bellinger, P. F., Christiansen, K. A. and Janssens, F. 1996-2021. Checklist of the Collembola of the World. Retrieved from https://www.collembola.org/.

Bellinger, P. F. and Ellis, W. N. 1997. Generic names of Collembola: Supplement 1984-1996. Tijdschrift voor Entomologie 140:1-11.

Cassagnau, P. and Deharveng, L. 1981. Sur le genre Vitronura (Collemboles: Neanuridae): aspect systématique et approache cytogénétique. Bulletin du Museum national d'histoire naturelle 3(1):151-173

Cassagnau, P. 1982. Sur les Neanurinae primitifs suceurs et les lignees qui en derivent (Collemboles). Travaux du Laboratoired' Ecobiologie des Arthropodes Edaphiques, Toulouse 3(3):1-11.

Cassagnau, P. 1984. Introduction al'etude des Phyiomeriens (Collemboles, Neanurinae): diagnoses preliminaires des especes. Travaux du Laboratoired' Ecobiologie des Arthropodes Edaphiques, Toulouse 4(3):1-30.

Cassagnau, P. 1993. Les Collemboles Neanurinae de I'Himalaya; III genre Nepalimeria. Revue Suisse de Zoologie 100(1):91-112. https://doi.org/10.5962/bhl.part.82502

Choudhuri, D. K. 1958. Some new species of Onychiurus Gervais (Collembola: Onychiuridae) from Nepal and Uganda. Proceedings of Royal Entomological Society of London, Series B 27:147-154. https://doi.org/10.1111/j.1365-3113.1958.tb00431.x

Christiansen, K. and Bellinger, P. 1992. Insects of Hawaii, Collembola. Honolulu, University of Hawaii press. p 445.

Christiansen, K. and Bellinger, P. 1996. Cave Pseudosinella and Oncopodura new to science. Journal of Caves and Karst Studies 58(1):38-53.

Cipola, N. G., Oliveira, F. G. L., Moraisi, J. W. and Bellini, B. C. 2016. The Heteromurini Absolon \& Ksenemann (Collembola, Entomobryidae): a review of the genera status and diagnoses, keys for species of Alloscopus Borner and Heteromurtrella Mari Mutt and description of a new species. Zootaxa 4084(2):151186. https://doi.org/10.11646/zootaxa.4084.2.1

D'Haese, C. and Weiner, W. M. 1998. A review of Willemia buddenbrocki group (Collembola, Poduromorpha, Hypogastruridae) with cladistics analysis. Journal of Natural History 32(7):969-986. https://doi.org/10.1080/00222939800770501

Deharveng, L. 1996. Soil Collembola diversity, endemism, and reforestation: A case study in the Pyrenees (France). Conservation Biology 10(1):74-84 https://doi.org/10.1046/j.1523-1739.1996.10010074.x

Deharveng, L., D'Haese, C. A. and Bedos, A. 2008. Global diversity of Springtails (Collembola: Hexapoda) in freshwater. Hydrobiologia 595:329-338. https://doi.org/10.1007/s10750007-9116-z

Deharveng, L., Palacios-Vargas, J. G. and Bedos, A. 2017. A list of Yuukianura Yosii, 1955 species of the world (Collembola: Neanuridae: Neanurinae: Lobellini), with description of a new species of unusual ecology from Santo Island (Vanuatu). Zoosystema 39(1):55-67. https://doi.org/10.5252/z2017n1a7

Dunger, W. and Voigtlander, K. 2009. Soil fauna (Lumbricidae, Collembola, Diplopoda and Chilopoda) as indicators of soil ecosubsystem development in post-mining sites of eastern Germany - a review. Soil Organisms 81(1):1-51.

Ellis, W. N. and Bellinger, P. F. 1973. An annotated list of the generic names of Collembola (Insecta) and their type species. Monografieen van de Nederlandse Entomologische Vereniging 7:1-74.

Ellis, W. N. and Bellinger, P. F. 1984. Generic names of Collembola: Supplement 1973-1983. Tijdschrift voor Entomologie 127:1-15. 
Fjellberg, A. 1977. On the identity of Isotoma ekmani nom. nov. pro I. pallida Agrell, 1939 (nec Nicolet, 1842, Moniez, 1894) (Collembola: Isotomidae). Scandinavian Entomology 8:9-11. https://doi.org/10.1163/187631277×00026

Folsom, J. W. 1902. Collembola of the grave. Psyche 9(315):363-367.

Frampton, G. K. 1994. Sampling to detect effects of pesticides on epigeal Collembola (Springtails). Aspects of Applied Biology 37:121-130.

Geissen, V. and Kampichler, C. 2004. Limits to the bioindication potential of Collembola in environmental impact analysis: a case study of forest soil-liming and fertilization. Biology and Fertility of Soils 39:383-390. https://doi.org/10.1007/s00374-003-0714-2

Greenslade, P. 1999. Revision of Folsomina Denis (Collembola: Isotomidae) with a description of a new species from Southeast Asia and the Pacific region and comments on ecology. The Raffles Bulletin of Zoology 47(1):1-15.

Greenslade, P. 2007. The potential of Collembola to act as indicators of landscape stress in Australia. Australian Journal of Experimental Agriculture 47:424-434. https://doi.org/10.1071/ea05264

Greenslade, P., Bell, L. and Florentine, S. 2011. Auditing revegetated catchments in southern Australia: decomposition rates and collembolan species assemblages. Soil Organisms 83(3):433450.

Gruss, I., Twardowski, J. P., Krolczyk, A. L. J. and MedynskaJuraszek, A. 2019. The effect of biochar used as soil amendment on morphological diversity of Collembola. Sustainability 11:5216.

Imms, A. D. 1912. On some Collembola from India, Burma and Ceylon; with a catalogue of the oriental species of the order. Proceedings of the General Meetings of Scientific Business of the Zoological Society of London, London, Messrs Longmans, Green, and Co.

Janetschek, F. 1990. Berichte des naturwissenschaftlichmedizinischenvereins in Innsbruck. Innsbruck, Universitatsverlag Wagner. p 119.

Jordana, R. and Baquero, E. 2005. A proposal of characters for taxonomic identification of Entomobrya species (Collembola: Entomobryomorpha), with description of a new species. Abhandlungen und Berichte des Naturkundesmuseums Gorlitz 76(2):117-134

Katz, A. D., Giordano, R. and Soto-Adames, F. 2015. Taxonomic review and phylogenetic analysis of fifteen North American Entomobrya (Collembola, Entomobryidae), including four new species. Zookeys 525:1-75.

https://doi.org/10.3897/zookeys.525.6020

Lawrence, P. N. 1969. Isotomidae from the Solomon Islands (Collembola). Pacific Insects 11(3-4):545-559.

Mandal, A. K. and Hazra, G. P. 2009. The diversity of Collembola (Hexapoda) from East and North-East India with some notes on their ecology. Records of the Zoological Survey of India, Occasional paper 298:1-206.

Mandal, G.P., Suman, K.K. and Bhattacharya K.K. 2010. On some collection of collembolan present in the apterygota section. Records of Zoological Survey of India 112(3):95-100.

Mandal, G.P. 2018. Collembola of India- An updated checklist. Halteres 9: 116-130. doi: 10.5281/zenodo. 1280640
Mari Mutt, J. A. 1979. A revision of genus Dicranocentrus Schött (Insecta: Collembola: Entomobryidae). M.Sc., University of Puerto Rico, Rio Piedras, Puerto Rico.

Mari Mutt, J. A. 1981. Two new Dicranocentrus from Nepal and a key to the Indian and Nepalese species (Collembola: Entomobryidae). Pan-Pacific Entomologist 57(4):493-599.

Mari Mutt, J. A. 1985. Eight species of Dicranocentrus and redescriptions for $D$. thaicus and $D$. pilosus (Collembola: Entomobryidae: Orchesellinae). Journal of Agriculture of University of Puerto Rico 69(3):297-322.

Mari Mutt, J. A. and Bhattacharjee, R. K. 1980. Four new species of Dicranocentrus from northeast India and Nepal (Collembola: Entomobryidae: Orchesellinae). Pacific Insects 22(1-2):162170.

Martín, J., García-Barros, E., Gurrea, P., Luciañez, M. J., Munguira, M. L., Sanz, M. J., Simón, J. C. 2000. High endemism areas in the Iberian Peninsula. Belgian Journal of Entomology 2:47-57.

Mitra, S. K. and Dallai, R. 1980. Studies of the genus Campylothorax SchÖtt, 1893 (Collembola: Entomobryidae: Paronellinae) with the description of a new species from Zaire. Italian Journal of Zoology 13(1):273-321.

Palacios-Vargas, J. G. and Gomez-Anaya, J. 1995. Two New Mexican Species of Paleonura (Collembola: Neanuridae). Journal of the Kansas Entomological Society 68(1):95-102.

Palacios-Vergas, J. G. and Deharveng, L. 2010. A new species of Kenyura (Collembola: Neanuridae: Pseudachorutinae) from Nicaragua. Brenesia 73-74:75-88.

Petrillo, H. A. and Witter, J. A. 2005. Invertebrate biodiversity in northern hardwood ecosystems under varying disturbance regimes. Proceedings of the Beech Bark Disease Symposium, Saranak Lake, NY, USA, Department of Agriculture, Forest Service, Northeastern Research Station.

Potapov, M. and Cassagnau, P. 2000. Two new species of Folsomia (Collembola, Isotomidae) from Nepal. Contributions from Biological Laboratory, Kyoto University 29(2):75-81.

Potapov, M., Janion, C. and Deharveng, L. 2011. Two new species of Parisotoma (Collembola: Isotomidae) from the Western Cape, South Africa. Zootaxa 2771:17-24. https://doi.org/10.11646/zootaxa.2771.1.2

Rodgers, D. J. and Kitching, R. L. 1998. Vertical stratification of rainforest Collembolan (Collembola: Insecta) assemblages: Description of ecological patterns and hypotheses concerning their generation. Ecography 21(4):392-400. https://doi.org/10.1111/j.1600-0587.1998.tb00404.x

Rodgers, D. J. and Kitching, R. L. 2011. Rainforest Collembola (Hexapoda: Collembola) and the insularity of epiphyte microhabitats. Insect Conservation and Diversity 4(2):99-106.

Rusek, J. 1998. Biodiversity of Collembola and their functional role in the ecosystem. Biodiversity \& Conservation 7(9):1207-1219. https://doi.org/10.1023/a:1008887817883

Santos, E. M. R., Franklin, E. and Luizao, F. J. 2008. Litter manipulation and associated invertebrate fauna in secondary forest, central Amazonia, Brazil. Acta Oecologica 34:274-284. https://doi.org/10.1016/j.actao.2008.05.011

Schulz, H.J. 2018. Bemerkenswerte Collembolenfunde aus Nepal (Insecta: Collembola).In Hartmann, M., M.V.L. Barclay \& J. Weipert (eds):Biodiversität und Naturausstattung im Himalaya 
VI- Verein der Freunnde und Förderer des Naturekundemuseums Erfurt e. V., Erfurt: 179-182.

Shaw, P. 2013. The use of inert pads to study the Collembola of suspended soils. Soil Organisms 85(1):69-74.

Skarzynski, D. and Smolis, A. 2006. Description of Ceratophysella robustiseta sp. n. from greenhouses in England, with notes on synonymy of $C$. postanetennalis Yosii, 1966 and taxonomic status of C. morula Deharveng \& Bourgeois, 1991 (Collembola: Hypogastruridae). Revue Suisse de Zoologie 113(2):297-303. https://doi.org/10.5962/bhl.part.80353

Snyder, B. A. and Callaham, M. A. 2019. Soil fauna and their potential responses to warmer soils. In: (Eds) Ecosystem consequences of soil warming. Elsevier Netherlands. pp 279-296. https://doi.org/10.1016/b978-0-12-813493-1.00012-0

Soto-Adames, F. N., Barra, J., Christiansen, K. and Jordana, R. 2008. Supragenic classification of Collembola Entomobryomorpha. Annals of Entomological Society of America 101(3): 501-513. https://doi.org/10.1603/0013-8746(2008)101[501:scoce]2.0.co;2

South, A. 1961. The taxonomy of the British species of Entomobrya (Collembola). Transections of the Royal Entomological Society of London 119:387-416. https://doi.org/10.1111/j.13652311.1961.tb00798.x

Tamura, H. and Zhao, L. 1997. Two Onychiurid species new to China (Collembola). Entomologia Sinica 4(1):47-52. https://doi.org/10.1111/j.1744-7917.1997.tb00070.x

Thapa, V. K. 1997. An inventory of Nepal's insects. Kathmandu, Nepal, IUCN-The World Conservation Union. p 475.

Thapa, V. K. 2015. Insect diversity in Nepal. Format Printing Press, Kathmandu, Nepal. p 1094.

Thibaud, J. M. and Najt, J. 1988. Collemboles (Insecta) de l'Equateur IV. Paronellidae avec revision de quatre genres. Bulletin du Museum National d'Histore Naturelle 10(4):719-730.

Timmermans, M., Roelofs, D., Mariën, J. and Van Straalen, N. 2008. Revealing pancrustacean relationships: Phylogenetic analysis of ribosomal protein genes places Collembola (springtails) in a monophyletic Hexapoda and reinforces the discrepancy between mitochondrial and nuclear DNA markers. BMC Evolutionary Biology 8(1):1-10. https://doi.org/10.1186/14712148-8-83

Vargovitsh, R. S. 2009. Species composition of the family Arrhopalitidae (Collembola: Symphypleona). Vestnik Zoologii 43(4):17-19. https://doi.org/10.2478/v10058-009-0016-8

Vázquez, M. M., Cutz-Pool, L. Q. and Palacios-Vargas, J. G. 1998. A new species of Hylaeanura (Collembola: Neanuridae: Pseudachorutinae). Southwestern Entomologist 23(4):367-371.

Wilson, J. M. 1982. A review of world Troglopedetini (Insecta, Collembola, Paronellidae) including an identification table and descriptions of new species. Transactions British Cave Research Association 9(3):210-226.

Yin, R., Eisenhauer, N., Schmidt, A., Gruss, I., Purahong, W., Siebert, J., et al. 2019. Climate change does not alter land-use effects on soil fauna communities. Applied Soil Ecology 140:1-10. https://doi.org/10.1016/j.apsoil.2019.03.026.

Yosii, R. 1959. Studies on the Collembolan faunaof Malay and Singapore with special reference to the genera Lobella, Lepidocyrtus and Callyntrura. Contributions from the Biological laboratory, Kyoto University 10:1-65.
Yosii, R. 1960. Studies on the Collembolan genus Hypogastrura. The American Midland Naturalist 64(2):257-281.

https://doi.org/10.2307/2422661

Yosii, R. 1962. Studies on the Collembolan genus Hypogastrura II. Contributions from the Biological Laboratory, Kyoto University 13:1-25.

Yosii, R. 1966a. On some Collembola of Afghanistan, India and Ceylon, collected by the Kuphe-expedition, 1960. Results of the Kyoto University Scientific Expedition to the Karakoram and Hindukush 8:333-405.

Yosii, R. 1966b. Collembola of Himalaya. Journal of the College of Arts and Sciences, Chiba University 4:461-531.

Yosii, R. 1969. Dicyrtomina and Ptenothrix (Insecta: Collembola) of the Solomon Islands. Zoological Journal of the Linnean Society 48(2):217-236.

https://doi.org/10.1111/j.1096-3642.1969.tb00712.x

Yosii, R. 1970. On some Collembola of Japan and adjacent countries II. Contributions from the Biological Laboratory, Kyoto University 23(1):1-32.

Yosii, R. 1971. Collembola of Khumbu Himal. Ergebn Forsch Unternehmens Nepal Himalaya 4(1):80-130.

Yosii, R. 1977. Critical check list of the Japanese species of Collembola. Contributions from the Biological Laboratory, Kyoto University 25(2):141-170.

Yosii, R. 1990. Report on the cryophilous Collembola, collected by Dr. S. Kohshima. Contributions from the Biological Laboratory, Kyoto University 27(4):523-534.

Zeppelini, D. 2011. Phylogeny of Arrhopalites s.I. (Collembola: Symphypleona: Arrhopalitidae): testing the monophyly of the recently erected genera Arrhopalites s.s. and Pygmarrhopalites. Invertebrate Systematics 25(2):91-105. https://doi.org/10.1071/is 10044

Zeppelini, D., Lopes, B. and Lima, E. 2019. A new species of Szeptyckitheca (Collembola, Symphypleona, Sphyrothecinae) from Brazil. Neotropical entomology 48(2):269-276.

Zhang, F. 2015. Some Willowsia from Nepal and Vietnam (Collembola: Entomobryidae) and description of one new species. Zootaxa 3905(4):489-499. https://doi.org/10.11646/zootaxa.3905.4.3

Zhang, F., Chen, J. X. and Deharveng, L. 2011. New insight into the systematics of the Willowsia complex (Collembola: Entomobryidae). Annales de la Société entomologique de France, Taylor \& Francis. https://doi.org/10.1080/00379271.2011.10697692 